\title{
¿TÚ O USTED? ESTIGMATIZACIÓN DEL TUTEO EN BOGOTÁ ${ }^{1}$ TÚ OR USTED? TUTEO STIGMA IN BOGOTÁ
}

\author{
Cristal Yeseidy Cepeda Ruiz \\ Universidad Nacional Autónoma de México \\ cristalcepedaruiz@comunidad.unam.mx
}

Resumen

En el presente artículo se analiza la influencia del factor social $s e x o^{2}$ (tanto del locutor como del interlocutor) en la selección de las formas de tratamiento pronominales tú y usted en la ciudad de Bogotá (Colombia); se estudia la asociación de tuteo con el habla femenina y el habla gay, específicamente, el rechazo de tú como trato entre hombres bogotanos y migrantes residentes en Bogotá, se propone que esta restricción pragmática ocasionada por el factor sexo favorece el uso mayoritario de usted e impide que el tuteo amplíe sus contextos de aplicación en Bogotá. Se presenta un análisis cuantitativo (frecuencias absolutas y relativas) / cualitativo (percepción y actitud de los hablantes) de los datos obtenidos de 36 cuestionarios sociolingüísticos, así como de fragmentos de la serie de televisión 'Betty la fea' (RCN televisión, 2010).

Palabras Clave: formas de tratamiento, poder, solidaridad, estigma y estereotipo social

\footnotetext{
${ }^{1}$ Este estudio forma parte de la investigación de maestría 'Usted, tú, sumercé y vos: formas pronominales de tratamiento en el español de Bogotá (Colombia)" (Cepeda, 2014) financiada por el Consejo Nacional de Ciencia y Tecnología (Conacyt). Agradezco a la doctora María Ángeles Soler Arechalde por la lectura de este artículo y sus acertadas correcciones.

${ }^{2}$ Considero fundamental iniciar este texto con la distinción, ya clásica en los estudios sociolingüísticos, entre sexo y género. El sexo es utilizado para indicar una distinción de tipo biológico que atañe a los seres vivos (plantas y animales) y que los clasifica en las categorías 'femenino' o 'masculino'; por su parte, el género se reserva para establecer un 'papel' o 'rol' a los individuos, ya no desde lo biológico sino como construcción sociocultural. Si bien no existe consenso pleno acerca de cuál factor utilizar y es recurrente encontrar investigaciones que prefieren una u otra categoría, en el presente artículo utilizaré el término sexo, pues tal como señala Blas Arroyo (2005), con este se incluye además de la distinción social, la biológica (el autor señala que no se ha demostrado plenamente que el componente biológico no esté relacionado de alguna manera con la variación lingüística).
} 
Abstract

This article analyses the influence of social factor gender (both the speaker and the interlocutor) in the selection of pronominal forms of address: tú and usted in Bogotá city (Colombia); We study the association of 'tuteo' with feminine speech and gay speech, specifically, the rejection of tú as address between Bogota's masculine citizens and migrants living in Bogota, we proposed that this pragmatic restriction caused by the gender factor favors the use of usted and prevents that 'tuteo' expands its application in different contexts in Bogota. The article shows a quantitative (absolute and relative frequencies) and qualitative (perception and speaker's attitudes) analysis of the data obtained from 36 sociolinguistic questionnaires, as well as fragments from the television series 'Betty la fea' (RCN television, 2010).

KEYWORDs: forms of address, power, solidarity, stigma, social stereotype

FECHA DE RECEPCIÓN: 24/10/2016

FECHA DE ACEPTACIÓN: 27/01/17

\section{Introducción}

Si bien los estudios sobre tratamientos pronominales señalan un incremento de las formas de confianza o cercanía - tú en las sociedades occidentales actuales (Brown y Gilman, 1960 y Fontanella, 1999), otro es el panorama que se observa en el español hablado en Bogotá (Colombia), ciudad en la que hoy en día predomina el uso de usted $^{3}$ (tratamiento prototípico de distancia) en casi todos los contextos: familia nuclear y extendida (1a), relaciones laborales (1b), intercambios con desconocidos (1c) y entre hombres (1d), quienes, como se verá más adelante, se ustedean ${ }^{4}$ con bastante frecuencia (Bartens, 2003 y Cepeda, 2014):

\footnotetext{
${ }^{3}$ Tal como en Costa Rica (Castillo, 2013), Guatemala (Hernández, 2013) y Colombia (Flores, 1977; Montes, 1985b; Carricaburo, 1997).

${ }^{4}$ El término 'ustedeo' es utilizado en la bibliografía especializada para definir dos fenómenos distintos; el primero es el uso, ampliamente difundido en países como Costa Rica (Quesada Pacheco, 1996; Moser, 2010a y 2010b; Castillo Venegas, 2013) y Colombia (Flórez, 1977 y 1980; Montes, 1985a y 1985b; Uber, 1985; Carricaburo, 1997; Cepeda, 2014), del pronombre usted en contextos íntimos, también conocido como usted solidario y usted-t (Bertolotti, 2015: 48). El segundo fenómeno se refiere al uso
} 
(1) ¿A qué persona prefiere tratar de "usted"?

a. CATALINA-M1BU: ${ }^{5}$ A mis papás.

b. LUISA-M2MU: A los empleados, por no dar mucha confianza.

c. SONIA M2BP: A desconocidos, porque no hay confianza con ellos.

d. GUSTAVO-H2AU: A los hombres y personas de mayor rango.

El tuteo, por su parte, se reserva en Bogotá como trato dado por - y dirigido a - mujeres (2a), niños (2b), jóvenes (2c) y entre individuos de clase alta (2d) (Flórez, 1980; Montes, 1985a; Bartens, 2003; Mestre, 2010 y Cepeda, 2014):

(2) ¿A quién tutea con facilidad?, ¿por qué?

a. JOSÉ-H1BU: A las mujeres, porque me gusta tratarlas bien a ellas.

b. LUISA-M2MU: A los niños, por ternura.

c. MARÍA-M1MU: Amigos, porque son de edades cercanas a la mía.

d. ALEX-H1AU: A todo el mundo.

El uso de tú en Bogotá se ve afectado por dos restricciones pragmáticas; en primer lugar, los bogotanos y migrantes consideran que el tuteo es un trato inadecuado en situaciones en las que no hay confianza con el interlocutor o hay diferencia de estatus desde una perspectiva moral o social (edad, jerarquía, etc.), en estos casos se considera que tú excede la confianza permitida y puede señalar incluso irrespeto ( $3 \mathrm{a}$ y b):

(3) ¿Le parece incómodo o inadecuado que lo tuteen? ¿En qué situaciones le parece incorrecto que lo tuteen? ¿Considera inapropiado que alguna(s) persona(s) lo tutee(n), quién(es)?

de usted como segunda persona singular independiente del contexto comunicativo, en ese sentido funciona como concepto análogo al de 'tuteo' (tratar de tú) y 'voseo' (tratar de $v o s)$. En estas páginas me decantaré por la segunda definición de ustedeo.

${ }^{5}$ El código de los informantes parte de un pseudónimo y las iniciales de cada variable social: sexo (hombre: $\mathbf{H}$, mujer: $\mathbf{M}$ ), generación (1ra: 1, 2da: 2, 3ra: 3), clase social (baja: B, media: M, alta: A) y lugar de origen (Bogotá: $\mathbf{U}$, lugar prototípicamente voseante/sumercedeante: $\mathbf{P}$, otra región del país: $\mathbf{R})$. 
a. VIOLETA-M1MU: Es difícil tutear a un adulto mayor, por el respeto que se merece, desde la casa enseñan que a personas de "ese tipo" se les debe hablar de usted.

b. LUIS-H2BU: Sería inapropiado hacerlo con superiores y con mayores de edad. Por respeto.

En segundo lugar, ciertos sectores de Bogotá (diatópicos, diastráticos, diageneracionales y diasexuales) ven el tuteo como un tratamiento 'delicado,' 'cariñoso', 'femenino', una forma propia de las mujeres y hombres 'afeminados' ${ }^{6}(4)$; en otras palabras, los informantes de este estudio, específicamente los hombres, comparten la creencia de que el habla gay es similar o comparte rasgos con el habla femenina, entre ellos el tuteo: ${ }^{7}$

(4) ¿Con cuál forma de tratamiento no se siente a gusto (dar y/o recibir)?

a. MANUEL-H2MU: No me gusta, me da mal genio que un hombre me tutee porque me siento degradado, gay, eso es propio de homosexuales.

b. HUGO-H3MU: Que me tutee un hombre, porque es de maricas.

Como consecuencia de la influencia del factor social sexo (además de otros como la edad y clase social), ${ }^{8}$ usted supera en frecuencia el uso de tú y posee menos restricciones de uso; en otras palabras, usted se ubica como tratamiento no marcado del español bogotano (Cepeda, 2014).

Las investigaciones previas suelen, por un lado, resaltar la alta frecuencia de usted en Bogotá, así como su presencia en contextos de distancia (desconocidos, superiores, etc.) y de cercanía

\footnotetext{
${ }^{6}$ El DLE define 'afeminado' como: "1: de un hombre: que en su persona, modo de hablar, acciones o adornos se parece a las mujeres., "3: Dicho de un hombre: homosexual."

${ }^{7}$ Esta marcación sexual del tuteo se observa en el español de Costa Rica, Guatemala, Honduras y Nicaragua (Carricaburo, 1997; Castro, 2001; Moser, 2010a y b; Chavarría, 2013).

${ }^{8}$ En este artículo presento los datos correspondientes a la variable social sexo. Para consultar los resultados concernientes al repertorio pronominal bogotano (incluyendo las formas vos y sumercé) véase Cepeda (2014).
} 
(parejas, amigos, etc.) y, por otro lado, repasar el papel de los jóvenes y las clases altas en la promoción del tuteo (Flórez, 1977 y 1980; Montes, 1985a y 1985b; Uber, 1985; Bartens, 2003).

En general, el estudio de las formas de tratamiento de Bogotá se ha centrado en el ustedeo y ha pasado por alto la complejidad y variación relacionada con el tuteo; la mira de los trabajos está puesta en el locutor, no en su interlocutor y mucho menos en la combinación de las variables sociolingüísticas de ambos participantes; finalmente, no suelen incluir las motivaciones sociales, psicológicas, lingüísticas, etc., que están detrás del uso/ desuso de una forma.

El objetivo de este estudio consiste en señalar las restricciones que impiden la pronta expansión de tú en Bogotá, mismas que resultan en el uso generalizado de usted. Se trata en todo caso de un cambio de perspectiva, que, como se verá más adelante, permite ver una relación entre ambos fenómenos.

La hipótesis señala la existencia de una limitación pragmática relacionada con el sexo del hablante y del oyente, misma que restringe el tuteo a las mujeres y homosexuales y ocasiona que usted se establezca como trato no marcado social y lingüísticamente.

El 'estigma', visto como una marca o comportamiento particular que hace al sujeto que lo posee objeto de repudio por parte de los miembros de su comunidad (Goffman, 2006) y el 'estereotipo social' o conducta lingüística enmarcada dentro de un grupo estigmatizado (Blas Arroyo, 2005) son conceptos fundamentales para comprender el fenómeno. Se propone aquí que el tuteo masculino es visto, especialmente por los varones, como un estigma que indica pertenencia al grupo homosexual, lo cual genera el rechazo, la crítica, la condena y, finalmente, el desuso de la forma tú entre hombres.

Los datos presentados provienen de dos fuentes. Primero, 36 cuestionarios sociolingüísticos aplicados a bogotanos y migrantes internos agrupados en cuotas según su sexo, edad y clase social; segundo, fragmentos extraídos de la serie 'Betty la fea' (RCN televisión, 2010). Se realizó un análisis cuantitativo, basado en la estadística descriptiva (frecuencias de uso absolu- 
tas y relativas) que se complementa con datos de percepción de los hablantes (recogidas en los cuestionarios) y fragmentos de la serie televisiva.

El artículo se desarrolla como sigue. Luego de esta introducción, el apartado II, antecedentes, presenta los aspectos básicos de la Teoría del poder y la solidaridad de Brown y Gilman (1960), se repasan allí las investigaciones sobre el tuteo y ustedeo bogotano, así como los pocos trabajos que vinculan el factor sexo con el rechazo de tratamientos pronominales. Posteriormente, el apartado III, metodología, incluye la delimitación de la muestra, los factores sociales de los colaboradores, así como la descripción de los materiales utilizados, sus ventajas y desventajas. Luego, el apartado IV presenta los resultados cuantitativos y cualitativos obtenidos en el estudio; en seguida, en el apartado $\mathrm{V}$, se ofrece una discusión a partir de los conceptos 'estigma' y 'estereotipo social'. Finalmente, se ofrecen las conclusiones del estudio y las referencias bibliográficas.

\section{Antecedentes}

Reciben el nombre de formas de tratamiento los pronombres de segunda persona singular y plural elegidos por el hablante para dirigirse a su interlocutor; ${ }^{9}$ la Nueva gramática de la lengua española señala que su elección depende de: "la confianza que exista entre los interlocutores, la cercanía, la solidaridad, la intimidad, el respeto, el nivel del que recibe el trato en relación con el de quien lo otorga, la situación comunicativa y su grado de formalidad, además de otros factores similares." (RAE-ASALE, 2009: 1250).

En efecto, los sistemas de tratamiento pronominales están estrechamente relacionados con factores sociales como la edad, clase social, el nivel educativo, origen y el sexo; así como con factores pragmáticos como el tema, canal, tipo de acto de habla, etc. (Blas, 1994; Hummel, Kluge y Vázquez, 2010; Bertolotti,

\footnotetext{
9 "Toda forma de tratamiento puede descomponerse en tres partes: elementos nominales, formas pronominales y formas verbales” (Calderón, 2010: 553).
} 
2015); de hecho, la variación presente en las formas de tratamiento está, la mayoría de las veces, condicionada por estos factores: "Dentro del conjunto de los signos lingüísticos, las formas y fórmulas de tratamiento pertenecen a los que más estrechamente se vinculan a la cultura y al comportamiento de una sociedad y a las actitudes de los hablantes hacia los interlocutores en las respectivas situaciones comunicativas. De ahí que tiendan a variar en todas las dimensiones de la lengua" (Hummel, Kluge y Vázquez: 2010, 15).

\section{a. Solidaridad y poder}

Según la propuesta de Brown y Gilman (1960), las formas de tratamiento permiten codificar dos dimensiones básicas de la vida en sociedad: poder y solidaridad. El poder surge en relaciones, generalmente, asimétricas en las que hay diferencias ideológicas, generacionales o de jerarquía moral, social, económica, entre los hablantes y se vincula con el eje vertical que establecen los interlocutores en su relación:

One person may be said to have power over another in the degree that he is able to control the behavior of the other. Power is a relationship between at least two persons, and it is nonreciprocal in the sense that both cannot have power in the same area of behavior. [...] There are many bases of power — physical strength, wealth, age, sex, institutionalized role in the church, the state, the army or within the family (Brown y Gilman, 1960: 255).

La solidaridad, por su parte, es simétrica y recíproca, es frecuente en relaciones que priorizan la igualdad, la pertenencia al mismo grupo religioso, político, de edad, de clase, etc., y generalmente se asocia con el eje horizontal: "If $A$ has the same parents as $B, B$ has the same parents as $A$. Solidarity is the name we give to the general relationship and solidarity is symmetrical. [...] The $T$ of solidarity can be produced by frequency of contact as well as by objective similarities" (Brown y Gilman, 1960: 258).

Los autores proponen que las lenguas especializan sus pronombres de tratamiento para designar respeto: formas $V$ y $f a-$ 
miliaridad: formas $T$ : "As a convenience we propose to use the symbols $T$ and $V$ (from the Latin $t u$ and vos) as generic designators for a familiar and polite pronoun in any language [...]. $T$ derives its common definition as the pronoun of either condescension or intimacy and $V$ its definition as the pronoun of reverence or formality" (Brown y Gilman, 1960: 254, 258).

El modelo considera que los tratamientos pronominales dependen de la semántica imperante en la relación entre locutor/interlocutor; en ese sentido, los ejes vertical y horizontal, a partir de rasgos como la edad, la clase social, el sexo, entre otros, determinan el uso de una forma $\mathrm{V}$ o T; las relaciones en las que predominan diferencias de poder se codifican mediante usos no recíprocos de $\mathrm{V}$ 'dar $\mathrm{T} /$ recibir $\mathrm{V}$ '; mientras que en las situaciones solidarias prevalecen usos recíprocos de formas $\mathrm{T}$.

Sin embargo, tal como mencionan varios autores (RAEASALE, 2009; Oliveira, 2010; Bertolotti, 2015, entre otros), el uso estricto de los términos 'confianza', 'respeto' 'familiaridad' no es siempre el más apropiado:

Siguen suponiendo [las investigaciones] que pueden aplicarse formas específicas a funciones específicas (por ejemplo, que el uso de una forma $\mathrm{V}$ indica formalidad o respeto y que una forma $\mathrm{T}$ indica informalidad o intimidad) y por lo tanto parecen ignorar que los hablantes sustituyen las interpretaciones convencionales de las formas con otras nuevas a la hora de negociar un patrón de tratamiento no convencional según las normas sociales (Oliveira, 2010: 60).

Más allá, parece que Brown y Gilman ven las relaciones sociales desde una perspectiva dicotómica; los autores consideran únicamente los extremos del continuum y descartan los diferentes grados de cercanía/distancia, confianza/respeto que negocian/renegocian los hablantes en su trato con otros a partir de la combinación de formas pronominales/verbales y fórmulas nominales (Rigatuso, 1994, citada por Fontanella, 1999; Calderón, 2010; Oliveira, 2010; Cepeda, 2014). ${ }^{10}$ A pesar de esto, la

${ }_{10} \mathrm{Tal}$ es el caso del sumercedeo bogotano (Cepeda, 2014; en prensa), tratamiento 
Teoría del poder y la solidaridad de Brown y Gilman continúa vigente y es el punto de partida de varias investigaciones sobre formas pronominales de tratamiento que se basan en sus planteamientos, pero incluyen una perspectiva mucho más dinámica de las relaciones sociales.

\section{b. Tú y usted en Bogotá}

El sistema de tratamientos del español de Bogotá no es dicotómico como lo es, por ejemplo, el de la Ciudad de México (Cepeda, en curso); además de las formas tú y usted, los bogotanos cuentan con los pronombres sumercé y vos (este último muy esporádico y asistemático) (Montes, 1985a; Cepeda, 2014). El uso de tres formas de tratamiento frecuentes para dirigirse al interlocutor, así como los diferentes valores pragmáticos y sociales que pueden asumir estos pronombres crean un sistema complejo: "En cuanto a los valores con que se usan los diversos pronombres, ello es buen indicio del estado conflictivo, un tanto caótico que actualmente tiene este subsistema en Bogotá. Casi cualquier valor puede asignarse a cualquier pronombre y sólo es posible asignar un valor predominante, pero no único, para cada forma pronominal" (Montes, 1985a: 306).

Sobre el uso de usted en Bogotá, Montes afirma que: “[...] es el más neutro en cuanto a valores, aunque con predominio del valor de distancia y respeto; sin embargo, sobre todo en estratos que usan poco el tú puede adoptar valores de confianza, amistad, afecto, etc." (1985a: 306); es decir, usted puede ser usado en contextos en los que predomina la solidaridad (contextos $\mathrm{T}$ ), así como en situaciones comunicativas en las que prevalece la distancia emocional, psicológica o física entre los hablantes (contextos V). El ustedeo predomina entre adultos, sujetos con nivel de estudio básicos (primaria o menos), entre la clase baja y media, especialmente de origen rural, los hombres y, finalmente, como trato asimétrico de inferiores a superiores, sea por edad

cuya semántica básica pertenece a la de una forma $\mathrm{V} / \mathrm{T}$, es decir, intermedia entre las formas $\mathrm{V}$ de respeto y $\mathrm{T}$ de intimidad. 
o por jerarquía (Rimgaila y Cristina, 1966; Flórez, 1980; Montes, 1985a; Uber, 1985; Montes et al., 1998; Bartens, 2003; Mestre, 2010 y Cepeda, 2014).

Por su parte, Cuervo (1867) señala que la sociedad bogotana de su época no tuteaba cotidianamente, sino que se reservaba este trato para usos literarios; esto nos permite apreciar que la forma ha expandido notablemente sus contextos de uso, puesto que ha dejado el plano literario para incorporarse en lo cotidiano. Con respecto a su significado de base, Montes (1985a) afirma que se trata de un valor de confianza, mientras que Uber (1985) asegura que el tuteo bogotano, a diferencia del peninsular, posee un significado intermedio entre la confianza y la distancia. Tú predomina en la clase alta, los jóvenes, sujetos con estudios superiores y las mujeres (Rimgaila y Cristina, 1966; Flórez, 1980; Montes, 1985a; Montes et al., 1998; Bartens, 2003; Mestre, 2010; Guerrero y Pardo, 2012; Cepeda, 2014). Por su parte, Flórez indica que el tuteo:

Se emplea cada vez más, sobre todo en los centros urbanos y entre gente joven, como tratamiento de confianza. En Bogotá hay ya muchos casos en que los inferiores - inclusive niños- tratan de tú a superiores en dignidad o en edad que apenas acaban de ver o de saludar (algunas personas consideran esta nueva práctica como falta de respeto) (1980: 34).

Este comportamiento le permite afirmar a Montes que: "dado que tú predomina en la juventud de todos los estratos, sobre todo en la de los altos, puede preverse que tal tratamiento continuará ganando terreno en el futuro inmediato" (Montes, 1985a: 307).

\section{c. Tratamiento 'estigmatizado'}

Si bien el sexo no suele ser una variable que determine de manera aislada el cambio lingüístico (Blas, 2005), en investigaciones previas se ha observado que, conjugado con otros factores como la edad o clase social, sí favorece la selección del tratamiento pronominal (Hummel, Kluge y Vázquez, 2010). 
Por su parte, el rechazo de un tratamiento debido al sexo del interlocutor es un fenómeno que ha sido reportado solo en algunas variedades del español: Costa Rica (Carricaburo, 1997; Moser, 2010a y b), Guatemala (Carricaburo, 1997; Moser, 2010a; Chavarría, 2013), Honduras (Castro, 2001), Nicaragua (Carricaburo, 1997) y Colombia (Bartens, 2003; Cepeda, 2014). Carricaburo, por ejemplo, señala que en Guatemala:

Con respecto al voseo hay un prejuicio sexista, un hombre cuando habla con otros recurre al voseo, pues el tuteo podría verse como afeminado e incluso como un síntoma de homosexualidad. En cambio, cuando se dirige a una mujer, puede optar por el vos o por el tú. En cuanto a la mujer, el empleo de vos se siente como vulgar o poco femenino (1997: 43).

Por su parte, Moser indica que en Honduras: "el sexo del hablante y de su interlocutor influye en la elección de la forma de tratamiento y consiste básicamente en que los hombres se manejan entre ellos con el voseo y las mujeres entre ellas con el usted o el tú, teniendo este último la marca de "homosexualidad" para los hombres (igual que en Costa Rica)" (2010a: 276).

En Bogotá, en una investigación realizada con estudiantes universitarios, Bartens encuentra que los hombres recurren al ustedeo para generar relaciones solidarias con otros hombres, mientras que las mujeres prefieren el tuteo:

Los hombres tienden a ustedear a otros hombres de su edad y situación social para crear un efecto de solidaridad o de confianza, mientras que tutean a mujeres de igual posición. Sin embargo, para asumir un tono de confianza con una amiga que habitualmente tutean, pueden ocasionalmente recurrir al ustedeo.

[...] Para las mujeres, el tuteo es el trato de más solidaridad y confianza. Algunas veces no lo brindan a hombres aún de posición social igual. Entonces el resultado es el trato no recíproco entre los dos sexos: los hombres tutean porque para ellos no es la forma de máxima confianza pero las mujeres no pueden tutear porque para ellas sí lo es (2003: 6-7). 
Bartens (2003) vincula el ustedeo y el tuteo a los conceptos de solidaridad y confianza; sin embargo, a diferencia de los estudios hechos en Costa Rica, Guatemala Honduras Nicaragua y Bogotá (Carricaburo, 1997; Castro, 2001; Moser, 2010a; Chavarría, 2013; Cepeda, 2014), la autora no encuentra una conexión con el desprestigio o rechazo de un tratamiento y el habla homosexual.

\section{Metodología}

\section{a. La muestra}

Por ser esta una investigación de corte sociolingüístico y al tratarse de un tema altamente vinculado con la realidad social, política y económica de la comunidad de habla estudiada, se hace necesario contar con una muestra que ejemplifique tal variedad (Lastra y Martín, 2000 y Orozco y Vázquez: 2010). Como consecuencia, la presente muestra recoge algunos de los rasgos sociales de los habitantes de Bogotá; se trata de una muestra 'estratificada no proporcional' asignada por cuotas ${ }^{11}$ equilibradas según los parámetros sexo: 18 hombres y 18 mujeres; clase social: baja (12), media (12) y alta (12); edad: 1ra generación - 15 a 34 años- (12), segunda generación — 35 a 49 años - (12) y tercera generación: - más de 50 años- (12). Además, se tuvo en cuenta el origen de los hablantes: bogotanos (27 individuos) y migrantes radicados en Bogotá ( 9 hablantes), en total 36 informantes.

Dos decisiones metodológicas concernientes a la muestra merecen ser descritas aquí. La primera corresponde al uso del factor 'clase social'; se trata de una variable ampliamente utilizada en trabajos sobre formas de tratamiento en Colombia (Rimgaila y Cristina, 1966; Flórez, 1977 y 1980; Montes, 1985a; Mon-

\footnotetext{
11 “[C]uotas establecidas para el número de personas que habrán de incluirse en las submuestras. Por ejemplo, tal vez los investigadores deseen un número igual de hombres y mujeres. Una vez que obtengan su cuota para un género [...]" (Clark-Carter, D. 2002, citado por Argibay, 2009: 18).
} 
tes et al., 1998; Cepeda, 2014, entre otros), ${ }^{12}$ debido a que todo colombiano es clasificado por el Estado en uno de seis estratos sociales. En ese sentido, el investigador no requiere crear, de manera artificial, un índice para catalogar a los hablantes en un número arbitrario de categorías. ${ }^{13}$ La 'clase social' es una variable de análisis eficaz puesto que está arraigada en el imaginario de la comunidad de habla estudiada ${ }^{14}$ y permite comparar los datos de investigaciones previas.

La segunda decisión atañe a la incorporación de migrantes en el presente estudio. Las investigaciones censales realizadas por el DANE (Departamento Administrativo Nacional de Estadística) señalan que durante el año 2005 Bogotá poseía un total de 6778691 habitantes, de estos, 38.6\% provenían de otros lugares de Colombia y del extranjero (DANE, 2010); es decir, Bogotá no es una ciudad homogénea en la que viven únicamente personas nacidas en su territorio, sino que es el punto de llegada de alrededor 2600000 migrantes que conviven diariamente. ${ }^{15}$ El 'origen' de los informantes permite examinar si el uso "estigmatizado de tú se extiende a otras zonas del territorio colombiano, específicamente permite apreciar si existe un proceso

\footnotetext{
${ }^{12}$ A pesar de ser un factor bastante criticado en las investigaciones de corte sociolingüístico (Blas, 2005).

${ }^{13}$ El objetivo de la clasificación consiste en suministrar apoyos económicos y sociales a los sujetos de clase baja (estratos 1 y 2), subsidiados por los miembros de clase alta 5 y 6. Se tiene en cuenta factores de la vivienda (ubicación, servicios, etc.) y de sus habitantes (nivel de ingresos y poder adquisitivo); en ese sentido, la estratificación se basa en la vivienda, pero se extrapola al sujeto que la habita. Véase http://www. dane.gov.co.

14 "La ley dice que son las residencias las que están estratificadas, pero se ha generalizado que se piense que lo que está estratificado son las personas" [...]. "Y esto se ha extendido a sus lugares de estudio, a los parques, a la manera de hablar" [consultado el 2 de febrero de 2017 de http://www.semana.com/vida-moderna/articulo/ estrato-1-estrato-6-como-los-colombianos-hablan-de-si-mismos-por-clases-sociales/403778-3.]

${ }^{15}$ Tal como lo señalan Lastra y Martín-Butragueño, toda investigación sociolingüística debe incluir la diversidad social presente en la comunidad de estudio, especialmente, en el caso de territorios en los que el contacto lingüístico es latente como Bogotá y la Ciudad de México: "Por todas estas razones el estudio de los inmigrantes está tan íntimamente enraizado en la comprensión sociolingüística de una ciudad como la de México. Sin ellos, el estudio no tiene sentido" (2000: 13).
} 
de acomodación lingüística de los tratamientos de los migrantes con el de sus anfitriones bogotanos" (Giles, 1984). Los migrantes incluidos en este estudio provienen de Boyacá, Meta, Santander, Quindío, Guaviare y Tolima y han residido en la ciudad entre 10 y 40 años.

\section{b. Las fuentes}

El cuestionario se basa principalmente en el utilizado por el equipo PRESEEA-Bogotá ${ }^{16}$ para extraer información sobre las formas pronominales de tratamiento del español de Bogotá (Molina, 2002). Sin embargo, la herramienta de esta investigación cuenta con más preguntas y no se limita a indagar por la forma que usa el locutor con $\mathrm{X}$ interlocutor en una situación $\mathrm{Y}$ o 'forma dada' (5), sino que incluye preguntas sobre la 'forma esperada' (6) o trato que el informante considera (o espera) recibir como oyente de ese mismo individuo (ahora en su papel como locutor); el objetivo de incluir estos dos tipos de preguntas consiste en analizar la direccionalidad del tratamiento, en otras palabras, constatar si el trato es recíproco o no: ${ }^{17}$

(5) ¿Cómo se dirige a su pareja cotidianamente (tú, usted, vos o sumercé)?, ¿hay alguna situación en que este tratamiento cambie?, ¿cuál es el nuevo tratamiento en esa situación?

(6) ¿Cómo se dirige su pareja hacia usted (tú, usted, vos o sumercé)?, ¿hay alguna situación en la que ese tratamiento varíe?, ¿cuál y qué tratamiento usa en esa nueva situación?

El cuestionario debe entenderse como: "una serie ordenada de preguntas con diferente grado de estructuración, susceptible

\footnotetext{
${ }^{16}$ El Proyecto PRESEEA tiene como objetivo: "la creación de un corpus de lengua española hablada representativo del mundo hispánico en su variedad geográfica y social" (http://preseea.linguas.net/). Para ello, los cerca de 40 equipos de trabajo utilizan una misma metodología y atienden a criterios sociolingüísticos para la recolección de los datos.

${ }^{17}$ En este documento detallaré únicamente los resultados obtenidos de las preguntas tipo 'forma dada'.
} 
de ser usado tanto en la interacción cara a cara como a distancia, y por lo general elaborado para ser aplicado a un grupo elevado de sujetos de manera individual o conjunta" (Paredes, 2010: 166); cuenta con seis secciones que dependen de la relación entre el locutor (encuestado) y su interlocutor: (1) Familia nuclear, (2) Familia extendida, (3) Amigos, (4) Trabajo/escuela, (5) Otros (profesiones, estrato y edad) y (6) Percepción de los tratamientos (valoraciones afectivas de los hablantes); las preguntas de las cinco primeras secciones son de tipo cerrado con cuatro opciones de respuesta correspondientes a los cuatro pronombres de tratamiento del español de Bogotá (usted, tú, sumercé y vos), mientras que la última parte del cuestionario (percepción) contiene preguntas abiertas.

Algunas desventajas de trabajar con cuestionarios, específicamente en investigaciones de corte sociolingüístico ${ }^{18}$ se relacionan con el carácter 'súper impuesto, 'artificial' y 'evaluativo' de estos instrumentos, lo que ha ocasionado que en múltiples ocasiones se cuestione la autenticidad de los datos y su correspondencia con la actuación de los hablantes (García Córdoba, 2002).

Por su parte, las ventajas de usar cuestionarios para extraer información sobre las formas de tratamiento de una comunidad son varias. Primero, permiten comparar los resultados de otras investigaciones; en ese sentido, podemos corroborar que la información extraída del instrumento es confiable (Paredes, 2010). Segundo, facilitan la recolección de datos cara a cara, de manera rápida y eficiente, a diferencia de otros métodos como la observación directa: "[...] permite consultar a una población amplia de una manera rápida y económica" (García Muñoz, 2003: 2). Tercero, por su carácter flexible, se puede adquirir tanta información como el investigador o el tema requieran y tanta como el informante esté dispuesto a ofrecer, en consecuencia, con el cuestionario se evita generalizar respuestas (García Muñoz, 2003: 2).

De otra parte, Betty la fea (RCN televisión, 2010) fue seleccionada como fuente secundaria de datos, esto con el objetivo

${ }^{18}$ Véase Orozco (2010). 
de incluir en la investigación información oral que se complementara con la extraída de los cuestionarios escritos.

El uso de este tipo de materiales en investigaciones de corte sociolingüístico, específicamente, las novelas literarias, ha sido cuestionado en repetidas ocasiones (Kany, 1976 y Labov, 1996) por tener un guion preestablecido y no corresponder al cien por ciento con el uso real de la lengua; sin embargo, en este estudio se presenta como una estrategia alternativa ante la imposibilidad de obtener datos orales completamente espontáneos como son las conversaciones no dirigidas.

Betty la fea se desarrolla en un contexto típicamente capitalino, presenta las vivencias de un grupo de trabajadores de clase baja y media en una prestigiosa empresa de modas bogotana -Ecomoda - y sus relaciones laborales/sentimentales con sus jefes de clase alta. Sus personajes son mayoritariamente bogotanos; sin embargo, la serie retrata bien la diversidad de Bogotá, hay migrantes de diferentes regiones del país (Antioquia, Valle del Cauca y Boyacá, entre otros) y del exterior (Argentina, Perú, Francia, etc.) y permite apreciar el uso de formas de tratamiento entre personas de diferente edad y clase social, así como entre esposos, vecinos, enemigos, desconocidos, etc.

Cabe resaltar que la serie no solo se escogió por situarse en Bogotá, sino que se incluyó la perspectiva de varios hablantes que consideraron que el habla, así como las situaciones que se presentan en Betty la fea, son un reflejo de la sociedad bogotana.

Finalmente, los datos extraídos de las cinco primeras secciones del cuestionario fueron analizados cuantitativamente por medio del programa estadístico GoldVarb X, esto permitió determinar el porcentaje de uso y la distribución de la variable dependiente: tú-usted, a partir de la variable independiente sexo. Por su parte, las respuestas de la última sección del cuestionario 'Percepción de los tratamientos' fueron decisivas para comprender la poca frecuencia de tú por parte de los hombres de la muestra; en ese sentido, el análisis cuantitativo se complementó con estas respuestas y se ejemplificó con fragmentos de la serie televisiva. 


\section{Resultados}

\section{a. Frecuencia y distribución de tú/usted}

A continuación, presento las frecuencias generales de uso de los tratamientos pronominales usted y tú reportados por los 36 informantes de la muestra en los cuestionarios sociolingüísticos (1350 datos). En el cuadro 1 el lector puede apreciar que usted es el trato más reportado por los informantes $(65.8 \%) ;{ }^{19}$ más allá, se infiere por los resultados que el tuteo no ha extendido sus contextos de uso como trato predominante, pues la diferencia porcentual entre ambos tratamientos pronominales es de $31.6 \%$ :

\begin{tabular}{|l|c|c|}
\hline PRONOMBRE & Frecuencia absoluta & Frecuencia relativa \\
\hline Usted & 888 & $65.8 \%$ \\
\hline Tú & 462 & $34.2 \%$ \\
\hline Total & 1350 & $100.0 \%$ \\
\hline
\end{tabular}

Cuadro 1. Uso general de las formas de tratamiento tú y usted en el español hablado en Bogotá

En el cuadro 2 se observa, tal como lo señalan investigaciones previas (Montes et al., 1998; Bartens, 2003), que el tuteo es más frecuente cuando el informante es mujer (57.1\%), mientras que el ustedeo es preferido por los hombres de la muestra (56.1\%):

\begin{tabular}{|l|c|c|c|c|c|}
\hline \multirow{3}{*}{ SeXo Trato } & \multicolumn{2}{|c|}{ Hombre } & \multicolumn{2}{c|}{ Mujer } & \\
\cline { 2 - 6 } & $F r$. & $F r$. & $F r$. & $F r$. & Total \\
& Tbs. & Rel. & Abs. & Rel. & \\
\hline Tú & 198 & $42.9 \%$ & 264 & $57.1 \%$ & $462 / 100.0 \%$ \\
\hline Usted & 498 & $56.1 \%$ & 390 & $43.9 \%$ & $888 / 100.0 \%$ \\
\hline
\end{tabular}

Cuadro 2. Forma de tratamiento dada por el informante según la variable sexo del locutor

\footnotetext{
${ }^{19}$ Los informantes de la muestra (hombres y mujeres) señalan con favoritismo el ustedeo en casi todos los contextos propuestos en el cuestionario (origen, clase social, edad, familia nuclear, amigos, trabajo, desconocidos, etc.), excepto con niños y con la pareja a quienes se tutea preferencialmente.
} 
Ahora bien, para algunos informantes el sexo del interlocutor es un rasgo relevante en la elección del trato que utilizan para dirigirse a su oyente (8):

(8) ¿Qué trato utiliza con sus primos?, ¿usa la misma forma para los primos maternos y para los paternos?

ALBERTO-H2MU: Tú a mujeres, usted a hombres.

En el cuadro 3 se encuentran estos casos en los que el encuestado especifica el sexo de su oyente, se trata de 129 tokens de los cuales 74 corresponden al uso de tú y 55 a usted; 67 respuestas pertenecen a los hombres y 62 a las mujeres. El lector puede apreciar que los encuestados tutean preferentemente a las mujeres (70.2\%); mientras que usan usted de manera predilecta con interlocutores hombres $(81.8 \%)$ :

\begin{tabular}{|c|c|c|c|c|c|}
\hline \multirow{2}{*}{ Trato } & \multicolumn{2}{|c|}{ Hombre } & \multicolumn{2}{|c|}{ Mujer } & \\
\hline & $\begin{array}{l}\text { Fr. } \\
\text { Abs. }\end{array}$ & $\begin{array}{l}\text { Fr. } \\
\text { Rel. }\end{array}$ & $\begin{array}{l}F r . \\
\text { Abs. }\end{array}$ & $\begin{array}{l}\text { Fr. } \\
\text { Rel. }\end{array}$ & Total \\
\hline Tú & 22 & $29.8 \%$ & 52 & $70.2 \%$ & $\begin{array}{c}74 / \\
100.0 \%\end{array}$ \\
\hline Usted & 45 & $81.8 \%$ & 10 & $18.2 \%$ & $\begin{array}{c}55 / \\
100.0 \% \\
\end{array}$ \\
\hline
\end{tabular}

Cuadro 3. Forma de tratamiento dada por el informante según la variable sexo del interlocutor

Los datos registrados en el cuadro 3 nos permiten observar el mismo patrón reportado en el cuadro 2, es decir, el tuteo predomina cuando se trata de mujeres y el ustedeo con los hombres, esta vez como oyentes.

Por otra parte, en el cuadro 4 se presenta el tratamiento reportado por el informante dependiendo de dos variables simultáneas: su sexo y el de su interlocutor; se incluyen en esta tabla únicamente las respuestas en las que el encuestado especifica el sexo de su oyente (129 datos). ${ }^{20}$

${ }^{20}$ Tal como se señaló para el cuadro 3, se trata de 129 tokens agrupados de la siguien- 
El lector puede apreciar que tú predomina como tratamiento en todos los casos en los que interviene una mujer (como locutor $\mathrm{u}$ oyente), especialmente de hombres a mujeres (60.8\%). En otras palabras, tú puede ser dado o recibido, en mayor o menor medida, por las mujeres.

\begin{tabular}{|l|c|c|c|c|c|}
\hline $\begin{array}{l}\text { SEXo } \\
\text { H/O }\end{array}$ & $\begin{array}{c}\text { Hombre a } \\
\text { hombre }\end{array}$ & $\begin{array}{c}\text { Hombre } \\
\text { a mujer }\end{array}$ & $\begin{array}{c}\text { Mujer a } \\
\text { mujer }\end{array}$ & $\begin{array}{c}\text { Mujer a } \\
\text { hombre }\end{array}$ & \\
\cline { 2 - 6 } TrATO & $\begin{array}{c}\text { Fr. Abs. / } \\
\text { Fr. Rel. }\end{array}$ & $\begin{array}{c}\text { Fr. Abs. / } \\
\text { Fr. Rel. }\end{array}$ & $\begin{array}{c}\text { Fr. Abs. } \\
/ \text { Fr. Rel. }\end{array}$ & $\begin{array}{c}\text { Fr. Abs. } \\
/ \text { Fr. Rel. }\end{array}$ & Total \\
\hline \multirow{2}{*}{ Tú } & 5 & 45 & 7 & 17 & 74 \\
\hline \multirow{2}{*}{ Usted } & $6.7 \%$ & $60.8 \%$ & $9.5 \%$ & $23.0 \%$ & $100.0 \%$ \\
\hline
\end{tabular}

Cuadro 4. Forma de tratamiento dada por el informante dependiendo de las variables sexo del locutor y sexo del interlocutor

A diferencia de lo que sucede cuando interviene una mujer, el tuteo entre hombres es muy bajo (cinco datos), en esta situación comunicativa se reporta con más frecuencia el uso de usted (34 casos); es decir, los hombres no suelen tutearse entre sí (6.7\%) y prefieren este trato para dirigirse a mujeres (60.8\%), tal como señala Bartens: "Los hombres tienden a ustedear a otros hombres de su edad y situación social para crear un efecto de solidaridad o de confianza, mientras que tutean a mujeres de igual posición" (2003: 6).

Los resultados cuantitativos vistos anteriormente permiten apreciar, primero, que en Bogotá existe un fuerte vínculo entre el pronombre de tratamiento y el sexo, no solo del informante, sino también de su interlocutor; ${ }^{21}$ segundo, tal como lo señalan

te manera: 67 datos reportados por los hombres y 62 por las mujeres, 74 de tuteo y 55 de ustedeo.

${ }^{21} \mathrm{El}$ análisis inferencial de GoldVarb reveló que la variable sexo es significativa y arrojó la siguiente jerarquización de factores para el ustedeo: '1. Sexo interlocutor 
otros autores, se reafirma que los bogotanos poseen un esquema de tratamientos frecuente, según el cual tú es usado por y para dirigirse a mujeres y usted entre hombres; tercero, se observa que, por un lado, las mujeres lideran y promueven el tuteo — variable lingüística innovadora-, y por otro lado, los hombres evitan el tuteo entre ellos (lo reservan para el trato con mujeres) e imponen el ustedeo — variable lingüística conservadora-.

Los datos cuantitativos presentados hasta el momento, por sí solos, no explican el comportamiento lingüístico y social de los bogotanos, sin embargo, sí nos permiten ver un patrón recurrente: el tuteo se suele vincular a las mujeres y es evitado por los hombres; asimismo podemos intuir que el uso de tú se ve restringido debido a la variable sexo (entre otras), en consecuencia, el ustedeo aparece en más contextos y es más frecuente.

\section{b. Percepción y actuación}

Los datos recolectados en la última sección del cuestionario (percepción de los tratamientos) permitieron entender el patrón detectado durante el análisis cuantitativo del estudio. Pudo apreciarse que quienes rechazan el uso de tú entre hombres pertenecen a grupos sociales con características similares entre sí; se trata de hombres ${ }^{22}$ de clase baja y media (muy pocos casos en la clase alta), bogotanos y migrantes de regiones ustedeantes de

$\mathbf{( 0 . 6 3 9 )}>2$. Sexo informante $(0.184)>3$. Edad informante $(0.161)>4$. Clase social informante (0.142)' (significance $=0.001 \mathrm{Log}$ likelihood $=-799.491$. Maximum possible likelihood $=-327.349$. Fit: X-square $(722)=944.284)$. Por su parte, los resultados inferenciales del tuteo indican la siguiente jerarquía: ' 1 . Sexo interlocutor $(0.666)$ $>2$. Origen informante $(0.617)>3$. Clase social informante $(0.330)>4$. Sexo informante (0.204) $>5$. Edad informante $(0.168)$ (significance $=0.000$ Log likelihood $=$ -614.717. Maximum possible likelihood $=-128.586$. Fit: $\mathrm{X}$-square $(923)=972.262)$.

22 Los hombres de la muestra son conscientes de este uso estigmatizado, a diferencia de lo que ocurre con las mujeres:

(a) ¿Con cuál forma de tratamiento no se siente a gusto (dar y/o recibir) tú, usted, sumercé o vos?

LEONARDO-H2AR: Tú con mi mismo género (dar y recibir. Hay una connotación sexual en Cúcuta con dicho empleo) [...] 
Colombia como Santander, Meta y Boyacá, ${ }^{23}$ mayoritariamente de segunda y tercera generación (9):

(9) ¿Le parece incómodo o inadecuado que lo tuteen? ¿En qué situaciones le parece incorrecto que lo tuteen? ¿Considera inapropiado que alguna(s) persona(s) lo tutee(n), quién(es)?

a. CAMILO-H1BU: Si es un hombre, sí.

b. HÉCTOR-H1MU: Incómodo que me tutee un hombre que ni lo conozca y se vea como raro (risas).

c. LUíS-H2BU: En general solo recibiría tuteo de mujeres demasiado cercanas, me parece incomodo que desconocidos lo hagan o que hombres de mi edad o mayores se dirijan a mí de esta forma.

d. IVÁN-H3BP: Cuando un hombre de mi edad o más joven me tutea, siento que eso es de las mujeres y de homosexuales.

Por el contrario, los hombres que no consideran inapropiado tutear a otros hombres pertenecen mayoritariamente a la clase social alta; esto se debe a que este grupo socioeconómico lidera el uso de tú (Rimgaila y Cristina, 1966; Montes, 1985a; Guerrero y Pardo, 2012; Cepeda, 2014), especialmente con miembros de su misma clase social; en ese sentido el tuteo de la clase alta introduce una marca de pertenencia al grupo; al parecer sus miembros priorizan la clase social ante otros factores como el sexo, la edad, etc. Ejemplos de este comportamiento pueden apreciarse en Betty la fea, en la serie es frecuente que los hombres de clase alta (dueños de Ecomoda) se tuteen entre ellos, sin reparar en la edad, el parentesco, tema de la conversación o el estado anímico de los participantes. En el siguiente fragmento se aprecia el uso de tú entre dos hombres de clase alta de segunda generación cuando están claramente enojados (10):

(10) Betty la fea: capítulo 016

- Daniel-H2AU: Tranquilos / no soy la muerte //

\footnotetext{
${ }^{23}$ En ese sentido, el fenómeno no es único del español bogotano, sino que se extiende a otras regiones de Colombia en las que predomina el ustedeo.
} 
- Armando-H2AU: No / eres como el novio de la muerte más o menos ¿no? / Daniel / y: cuéntame / ¿a qué se debe el placer de tu visita? //

- Daniel-H2AU: Quisiera tener el gusto de leer el informe que vas a presentar mañana en la junta / en medio del chismorreo / la discusión / y el almuerzo / no es fácil leer un informe que requiere de tanta atención //

- Armando-H2AU: Hombre / Daniel / yo no voy a poder darte ese gusto / figúrate / porque el informe solo te lo voy a entregar / ¡a la hora que te lo tenga que entregar! //

No obstante, en la serie se observa que el tuteo entre hombres se evita cuando existen diferencias relacionadas con la clase social y con individuos que son abiertamente homosexuales. Este último es el caso de Hugo Lombardi, diseñador de modas gay que se insinúa frecuentemente a los hombres de la empresa que tienen su misma edad y posición social (en especial con $\mathrm{Ar}$ mando y Mario); Hugo tutea a Mario y a Armando, pero solo es tuteado por este último; Mario, quien suele tutear a Armando y a otros hombres de su misma clase social, rechaza las insinuaciones de Hugo tratándolo de usted (11): ${ }^{24}$

(11) Betty la fea: capítulo 038

- Armando-H2AU: Hugo / estamos comprando telas 30\% poliéster / 70\% algodón / tú sabes que en este momento no podemos comprar 100\% algodón / ¡entiéndelo! //

- Hugo-H2AU: Es que no se trata del 70-30 / se trata de que la producción de las telas bajó / y después me dicen a mí que por qué las ventas están bajando //

- Mario-H2AU: ¡Ay / Hugo! / deje la menopausia precoz / que tampoco ha sido tan fuerte la incidencia de las telas / ipor dios! //

De hecho, tanto en las actitudes de los personajes masculinos (heterosexuales) de la serie Betty la fea, ${ }^{25}$ como en las

\footnotetext{
${ }^{24}$ En la serie se observa la tensión que existe entre Mario, el 'macho alfa' de la empresa y Hugo, una figura pública reconocida por su homosexualidad.

${ }^{25}<$ https://www.youtube.com/watch?v=CuRJfqQwlZ8>
} 
respuestas de percepción del cuestionario pudo apreciarse que los bogotanos y migrantes residentes en Bogotá no solo tienen objeciones acerca de usar tú como trato con otros hombres, sino que consideran 'incómodo', incluso 'ofensivo', que otros hombres, especialmente los homosexuales, los tuteen (12), por lo cual prefieren usar usted, especialmente cuando existe una marca (verbal/no verbal) que confiera al hablante/oyente como homosexual (13):

(12) ¿Con cuál forma de tratamiento no se siente a gusto (dar y/o recibir) tú, usted, sumercé o vos?

GUSTAVO-H2AU: Me incomoda que los gays me tuteen y no es por discriminación.

(13) Fragmento de novela Betty la fea: capítulo 038

- Maquillador-H2: ¡Listo! / ya puedes irte / ¡quedaste regia! //

- Armando-H2AU: ¡Umm! / ¿qué? / ¿qué hice yo para merecerme esto! / ¿ah! / ¿por qué vestido así! / ¡no puedo salir yo! / ¿Usted qué mira? //

- Maquillador-H2: ¡Perdón! //

\section{Discusión}

El uso sexualmente marcado de los tratamientos pronominales debe ser examinado, a mi juicio, a partir de dos conceptos propuestos por la sociología y la sociolingüística: el estigma y el estereotipo social.

Goffman define el estigma como: "un atributo profundamente desacreditador [...]; una clase especial de relación entre atributo y estereotipo" (2006: 14); el autor propone tres clases de estigmas: a. Los asociados a deformaciones del cuerpo, b. Los relacionados con el carácter del individuo, y c. Los vinculados con temas como la raza, la religión, la nación, etc. Para Goffman la consecuencia inmediata del estigma es el rechazo, que puede manifestarse mediante actitudes verbales (y no verbales) con las cuales los sujetos 'normales' - "[...] todos aquellos que no se 
apartan negativamente de las expectativas particulares que están en discusión"- (2006: 15) se alejan o segregan a los individuos estigmatizados: "en nuestro discurso cotidiano utilizamos como fuente de metáforas e imágenes términos específicamente referidos al estigma, tales como inválido, bastardo y tarado, sin acordarnos, por lo general de su significado real" (2006: 15).

Por su parte, Blas define el estereotipo social como todos aquellos: "rasgos definitorios del habla de ciertos grupos sociales con escaso prestigio social (clases bajas, grupos étnicos marginados, etc.) [...] otra característica esencial de los estereotipos - en la práctica derivada de la anterior - es su alto grado de estigmatización social en el seno de la comunidad de habla, lo que eventualmente puede conducir a su desaparición" (2005: 143).

La comunidad puede ser consciente de que $\mathrm{X}$ rasgo es un estereotipo de $\mathrm{Z}$ grupo, es decir, puede reflexionar acerca de la existencia del estereotipo; sin embargo, es común que pasen desapercibidos por los hablantes ${ }^{26}$ y que para su identificación se requieran pruebas de actitud lingüística (Blas, 2005).

Ciertamente la distinción - tuteo con mujeres y ustedeo con hombres - es un reflejo de la visión de mundo de los bogotanos, una visión que es, entre otras cosas, sexista (West y Zimmerman, 1975; Eckert, 1997; Lemus, 2001), en ese sentido, los resultados cuantitativos y cualitativos de esta investigación permiten deducir que los bogotanos y migrantes cuestionan el comportamiento de los hombres que tienen preferencias sexuales por su mismo sexo; en otras palabras, los hombres heterosexuales de la muestra consideran que los homosexuales se alejan de lo que la sociedad asigna como 'norma', la diferencia justifica la estigmatización y, en consecuencia, la exclusión del grupo mediante estrategias de distanciamiento social/verbal como el ustedeo.

Dos inferencias se derivan del escaso uso de tuteo entre hombres observado en el cuadro 4, así como en las respuestas de percepción. Por un lado, se aprecia que los bogotanos asumen que el habla de los hombres homosexuales comparte rasgos con

\footnotetext{
${ }^{26}$ Como en el caso de las bogotanas y mujeres migrantes que no son conscientes de este estereotipo.
} 
el de las mujeres; el estereotipo consiste en asumir que el uso de formas de cariño o confianza es típico del 'habla delicada' de las mujeres y homosexuales.

Por otro lado, siguiendo el concepto de 'estigma' de Goffman (2006), vemos cómo los tratamientos se convierten en una estrategia lingüística del bogotano para alejarse/distinguirse del homosexual estigmatizado. Bosque señala al respecto que: "[...] el lenguaje puede usarse, en efecto, con múltiples propósitos. Puede emplearse para describir, ordenar, preguntar, ensalzar o insultar, entre otras muchas acciones, y, desde luego, también puede usarse para discriminar a personas o a grupos sociales" (2010: 3).

Considero prudente señalar que este artículo no apoya la idea (bastante frecuente por estos días) de una 'lengua sexista' (en todo caso deberíamos hablar de individuos sexistas), tampoco propongo en estas páginas una política lingüística feminista que procure la erradicación de la distinción que hacen los bogotanos para tratar a hombres y mujeres, ni la resignificación de las formas de tratamiento en el habla bogotana, pues, como señalé anteriormente, se trata de un reflejo de la visión de mundo de la comunidad estudiada; es decir, lo que manifiesta este uso estigmatizado es que estamos frente a una sociedad altamente jerarquizada (edad, clase social, sexo, etc.), en la que se priorizan ciertas categorías frente a otras (alta/baja, hombre/ mujer, mayor/menor, etc.).

\section{Conclusiones}

En el presente artículo analizamos la relación del factor social sexo con la elección y rechazo de las formas de tratamiento tú/ usted en el español hablado en Bogotá. Pudimos apreciar, a partir de los resultados cuantitativos y cualitativos de esta investigación, que existe un esquema que se repite de manera frecuente: tú predomina con las mujeres y usted con los hombres.

Asimismo, los resultados permiten afirmar que el ustedeo es el tratamiento no marcado del español hablado en Bogotá y que el tuteo posee restricciones pragmáticas asociadas al factor 
sexo (entre otras), que restringen su uso y expansión; en ese sentido, el hecho de que los hombres encuestados consideren que tutear a otros hombres es una muestra de 'debilidad', 'femineidad' y 'homosexualidad' impide que este trato ocupe los mismos contextos de los que goza el pronombre usted, tratamiento que es visto por los sujetos de la muestra como el más utilizado en Bogotá y el que menos inconvenientes sociales y lingüísticos ${ }^{27}$ les ocasiona.

Finalmente se pudo apreciar que los hombres de este estudio opinan que tú, trato prototípico de las relaciones de confianza y cercanía, es propio del habla femenina y del habla gay; mientras que consideran que usted, tratamiento de respeto y distancia, pertenece al habla masculina. En consecuencia, los varones encuestados en esta investigación seleccionan el pronombre de tratamiento según tres distinciones relacionadas con el sexo de su interlocutor: i. hombres (+ USTED), ii. Mujeres (+TÚ) y iii. homosexuales (+USTED) y consideran que los hombres homosexuales y las mujeres usan el mismo repertorio pronominal (+TÚ).

\section{Bibliografía}

Argibay, Juan Carlos (2009), "Muestra en investigación cuantitativa", Subjetividad y Procesos Cognitivos, 13, 1, pp. 13-29. Bartens, Ángela (2003), Notas sobre el uso de las formas de tratamiento en el español colombiano actual. Comunicación presentada en el coloquio de Pronoms de 2 personne et forms d'adresse dans les Langues d'Europe, 7 a 8 de marzo de 2003, París, Instituto Cervantes [en línea], Centro virtual Cervantes: <http://cvc.cervantes.es/obref/coloquio_paris/ ponencias /baryens.htm> [consultado el 5 de noviembre de 2013].

\footnotetext{
${ }^{27}$ Un fenómeno recurrente en Bogotá es el uso de formas que alternan entre el tuteo y el ustedeo a nivel de palabra 'cómatelo’ y oración ‘¿se pegaste duro?' (Rimgaila y Cristina, 1966; Flórez, 1977; Guerrero y Pardo, 2012 y Cepeda, 2014).
} 
Bertolotti, Virginia (2015), A mí de vos no me trata ni usted ni nadie. Sistemas e historia de las formas de tratamiento en la lengua española en América, México, Universidad Nacional Autónoma de México/Universidad de la República Uruguay.

Blas Arroyo, José Luis (1994), "Los pronombres de tratamiento y la cortesía", Revista de Filología de la Universidad de La Laguna, 13, pp. 7-35.

Blas Arroyo, José Luis (2005), Sociolingüística del español. Desarrollos y perspectivas en el estudio de la lengua española en contexto social, Madrid, Cátedra.

Bosque, Ignacio (2010), Sexismo lingüístico y visibilidad de la mujer, Madrid, Real Academia Española.

Brown, Roger y Albert Gilman (1960), “The pronouns of power and solidarity", en T. Sebeok (ed.), Style in Language, Cambridge, MIT Press, pp. 253-276.

Calderón Campos, Miguel (2010), "Los elementos nominales en el sistema de tratamientos del español de Andalucía durante la restauración (1875-1931)", en M. Hummel, B. Kluge y M. E. Vázquez Laslop (eds.), Formas y fórmulas de tratamiento en el mundo hispánico, México, El Colegio de México, Karl Franzens Universität, pp. 551-570.

Carricaburo, Norma (1997), Las fórmulas de tratamiento en el español actual, Madrid, Arco Libros.

Castillo Venegas, María de los Ángeles (2013), “El español de Costa Rica: nivel morfosintáctico”, en M. Á Quesada Pacheco (ed.), El español hablado en América Central: nivel morfosintáctico, Vervuert, Iberoamericana, pp. 297-339.

Castro, Amanda (2001), Los pronombres de tratamiento en el español de Honduras, Múnich, Lincom Europe.

Chavarría Úbeda, Carmen (2013), "El español de Guatemala: nivel morfosintáctico", en M. Á Quesada Pacheco (ed.), El español hablado en América Central: nivel morfosintáctico, Vervuert, Iberoamericana, pp. 65-140.

Cepeda Ruiz, Cristal Yeseidy (2014), Usted, tú, sumercé y vos: formas pronominales de tratamiento en el español de Bogotá (Colombia), tesis de maestría, México, Universidad Nacio- 
nal Autónoma de México, en <http://bc.unam.mx/indexalterno.html>

Cepeda Ruiz, Cristal Yeseidy (en prensa), "El uso bogotano del tratamiento pronominal sumercé”, en J. C. Serrano y M. Á Soler Arechalde (eds.), Contacto lingüístico y contexto social. Estudios de variación y cambio, IV Coloquio Internacional de Cambio y Variación Lingüística, Contacto Lingüístico, México, Universidad Nacional Autónoma de México.

Cuervo, Rufino José (1867), Apuntaciones críticas sobre el español bogotano con frecuente referencia al de los países de hispano-américa, Bogotá, Instituto Caro y Cuervo.

Eckert, Penelope (1997), "Gender and sociolinguistic variation", en J. Coates (ed.), Readings in Language and Gender, Oxford, Blackwell.

DANE (2010), Informe general censo 2005, Bogotá, DANE.

Flórez, Luis (1977), Apuntes de español, Bogotá, Instituto Caro y Cuervo.

FLóreZ, LuIs (1980), "Datos de morfología y habla culta informal bogotana", Thesaurus, XXXV, 1, pp. 1-79.

Fontanella De Weinberb, María Beatriz (1999), "Sistemas pronominales de tratamiento usados en el mundo hispánico", en I. Bosque y V. Demonte (dirs.), Gramática descriptiva de la lengua española, Madrid, Espasa, 3, pp. 1399-1426. García Córdoba, Fernando (2002), El cuestionario. Recomendaciones metodológicas para el diseño de cuestionario, México, Limusa.

GARcía MuÑoz, Tomás (2003), El cuestionario como instrumento de investigación/evaluación, [en línea] <https://www. uam.es/personal_pdi/stmaria/jmurillo/Met_Inves_Avan/ Presenta ciones/Cuestionario_(trab).pdf> [consultado el 22 de agosto 2013].

Giles, HowArd (1984), “The dynamics of speech acommodation", International Journal of the Sociology of Languaje, 47, pp. 5-32.

Goffman, Erving (2006), Estigma: la identidad deteriorada, Buenos Aires, Amorrortu. 
Guerrero Rivera, Javier y Néstor Pardo (2012), “La fórmula de tratamiento sumercé, una aproximación preliminar desde la sociolingüística”, en C. P. Roselli y J. B. León Gómez (eds.), El lenguaje en Colombia: Realidad lingüística de Colombia, Bogotá, Academia Colombiana de la Lengua-Instituto Caro y Cuervo, tomo 1, pp. 211-218.

Hernández Torres, Ramón A. (2013), "El español de Honduras: nivel morfosintáctico”, en M. Á. Quesada Pacheco (ed.), El español hablado en América Central: nivel morfosintáctico, Vervuert, Iberoamericana, pp. 191-223.

Hummel, Martin, Bettina Kluge y María Elena Vásquez LASLOP (eds.). (2010), Formas y fórmulas de tratamiento en el mundo hispánico, México, El Colegio de México, Karl Franzens Universität.

Kany, Charles E. (1976), Sintaxis hispanoamericana, Madrid, Gredos.

Labov, William (1996), Principios del cambio lingüístico. 1: Factores internos, Madrid, Gredos.

Lastra, Yolanda y Pedro Martín Butragueño (coords.) (2000), "El modo de vida como variable sociolingüística en el estudio de la ciudad de México", en P. Martín Butragueño (ed.), Estructuras en contexto. Estudios de variación lingüística, México, El Colegio de México, pp. 13-43.

Lemus, Jorge (2001), "Sexismo en el lenguaje: mitos y realidades", en Memorias del Encuentro de la Red Centroamericana de Antropología, San Salvador, Asociación Salvadoreña de Antropología, pp. 195-225.

Mestre Moreno, Pilar (2010), “Alternancia de formas de tratamiento como estrategia discursiva en conversaciones colombianas", en M. Hummel, B. Kluge y M. E. Vázquez Laslop (eds.), Formas y fórmulas de tratamiento en el mundo hispánico, México, El Colegio de México, Karl Franzens Universität, pp. 1033-1050.

Molina Martos, Isabel (2002), Cuestionario sobre las formas de tratamiento, Bogotá, PRESSEA.

Montes Giraldo, José JoAquín (1985a), “El español bogotano 
en 1983 muestra fonética y gramatical", THESAURUS, XL, 2, pp. 294-307.

Montes Giraldo, José JoAquín (1985b), Estudios sobre el español de Colombia, Bogotá, Instituto Caro y Cuervo.

Montes Giraldo, José Joaquín, Gloria Duarte Huertas, María Bernarda Espejo Olaya, Jennie Figueroa Lorza, Mariano Lozano Ramírez, Siervo Mora Monroy Y Ricardo Ramírez Caro (1998), El español hablado en Bogotá, Análisis previo de su estratificación social, Bogotá, Instituto Caro y Cuervo.

Moser, Karolin (2010a), "Las formas de tratamiento verbalespronominales en Guatemala, El Salvador, Panamá (y Costa Rica): hacia una nueva sistematización en la periferia centroamericana”, en M. B. Hummel, Kluge y M. E. Vázquez Laslop (eds.), Formas y fórmulas de tratamiento en el mundo hispánico, México, El Colegio de México, Karl Franzens Universität, pp. 271-291.

Moser, Karolin (2010b), "San José (Costa Rica): desde los dignificados pragmáticos del ustedeo en el registro coloquial actual hacia sus primeras manifestaciones en el valle central (siglo XVIII)", en M. B. Hummel, Kluge y M. E. Vázquez Laslop (eds.), Formas y fórmulas de tratamiento en el mundo hispánico, México, El Colegio de México, Karl Franzens Universität, pp. 671-713.

Oliveira, Sandi Michelle de (2010), "La integración de la teoría y la metodología como desencadenante de un nuevo modelo de formas y fórmulas de tratamiento", en M. Hummel, B. Kluge y M. E. Vázquez Laslop (eds.), Formas y fórmulas de tratamiento en el mundo hispánico, México, El Colegio de México/Karl Franzens Universität, pp. 57-77. Orozco Vaca, María Leonor (2010), Estudio sociolingüístico de la cortesía en tratamientos y peticiones. Datos de Guadalajara, Tesis de doctorado no publicada, P. Martín-Butragueño (asesor), México, Colegio de México, Centro de Estudios Lingüísticos y Literarios.

Orozco Vaca, María Leonor y María Eugenia Vázquez Laslop, (2010), "Formas de tratamiento del español de 
México", M. B. Hummel, Kluge y M. E. Vázquez Laslop (eds.), en Formas y fórmulas de tratamiento en el mundo hispánico, México, El Colegio de México, Karl Franzens Universität, pp. 248-269.

Paredes, Florentino (2010), “¿Es factible un cuestionario estándar para el estudio del tratamiento? La experiencia del proyecto PRESEEA en Madrid y Alcalá de Henares", en M. Hummel, B. Kluge y M. E. Vázquez Laslop (eds.), Formas y fórmulas de tratamiento en el mundo hispánico, México, El Colegio de México/Karl Franzens Universität, pp. 164 $-191$.

Quesada Pacheco, Miguel Ángel (1996), “El español de América Central”, en M. Alvar (ed.), Manual de dialectología hispánica: El español de América, Barcelona, Ariel, pp. 101-115.

Real Academia Española-Asociación de Academias de LA Lengua (2009), "Las formas de tratamiento", en Nueva gramática de la lengua española, Madrid, Espasa, Tomo 1, pp. 1250-1268.

Rimgaila, Bárbara y María Teresa Cristina (1966), "La familia, el ciclo de vida y algunas observaciones sobre el habla en Bogotá", THESAURUS, 21, pp. 551-649.

Uber, Diane Ringer (1985), “The Dual Function of usted: Forms of Address in Bogotá, Colombia", Hispania, 68, pp. 388-392.

West, Candance y Don Zimmerman (1975), "Sex roles, interruptions and silences in conversation", en B. Thorne y N. Henley (eds.), Language and sex: difference and dominance, Rowley, Newbury House, pp. 105-129. 
